\title{
Tempo de latência e exatidão para leitura e nomeação em crianças escolares: estudo piloto*
}

\author{
Luciene Stivanin \\ Claudia Inês Scheuer \\ Universidade de São Paulo
}

\section{Resumo}

O objetivo deste estudo foi verificar o tempo de latência e o número de acertos para estímulos equivalentes em nomeação de figuras e leitura de palavras isoladas em voz alta, considerando os atributos do material escrito (freqüência de ocorrência e extensão da palavra). Os participantes foram crianças de $2^{\text {a }}$ a $4^{\text {a }}$ séries do ensino fundamental de uma escola pública e municipal da cidade de São Paulo, sem alterações gerais de desenvolvimento, de linguagem e escolares. A prova de leitura foi constituída por 32 palavras, balanceadas por freqüência de ocorrência no material escrito e extensão da palavra. A prova de nomeação foi constituída por figuras com nomes equivalentes às palavras da prova de leitura. Os estímulos foram apresentados individualmente na tela de um computador com software específico para análise do tempo de latência e das respostas. Foram analisados o número de acertos e o tempo de latência para as respostas. Para o tempo de latência, foi observada a influência das variáveis do material escrito, ou seja, palavras de baixa freqüência e de maior extensão foram acessadas mais lentamente por todas as crianças. Quanto ao número de respostas corretas, não houve diferença estatística entre as séries escolares na prova de leitura. Porém, com a idade e a escolarização, as crianças tornam-se mais rápidas para iniciar a leitura de palavras isoladas e para nomear figuras equivalentes.

\section{Palavras-chave}

Desenvolvimento - Tempo de latência - Leitura - Nomeação.

Correspondência:

Luciene Stivanin

Rua Teodoro Sampaio, 632, ap. 52

05406-000 - São Paulo - SP

e-mail: stivanin@usp.br

* Apoio financeiro da Fundação de Amparo à Pesquisa do Estado de São Paulo (processo 03/10036-0). 


\title{
Latency time and accuracy at reading and naming in school children: a pilot study ${ }^{*}$
}

\author{
Luciene Stivanin \\ Claudia Inês Scheuer \\ Universidade de São Paulo
}

\begin{abstract}
The objective of this study was to observe the latency time and the number of right answers for equivalent stimuli in naming pictures and in the reading aloud of isolated words, considering the attributes of the written material (frequency of occurrence and word length). The participants were pupils from the $2^{\text {nd }}$ to $4^{\text {th }}$ grades of the fundamental education of a public municipal school of the City of São Paulo; pupils had no general alterations in their development, language or school performance. The reading test was composed of 32 words balanced for frequency of occurrence in the written material and word length. The naming test consisted of pictures with names equivalent to those of the reading test. The stimuli were presented individually on the screen of a computer by a piece of software specific for the analysis of the latency time and accuracy of answers. The number of right answers and the latency time for response were analyzed. For the latency time, it was observed the influence of the variables of the written material, namely, that longer or low frequency words were accessed more slowly by all children. As to the number of right answers, there was no statistical difference between the grades at the reading test. However, with age and schooling, pupils become quicker to initiate the reading of isolated words and to name equivalent pictures.
\end{abstract}

\section{Keywords}

Luciene Stivanin

Rua Teodoro Sampaio, 632, ap. 52

05406-000 - São Paulo - SP

e-mail: stivanin@usp.br

Development - Latency time - Reading - Naming.

*This study was supported by FAPESP (The State of São Paulo Research Funding Agen-cy) under project n. 03/10036-0. 


\section{Introdução}

Nomear figuras e ler palavras escritas é uma das formas de investigar como informações lingüísticas vão sendo armazenadas ao longo do desenvolvimento e como podem ser recuperadas diante de um estímulo.

Assim, em relação à nomeação de figuras, o processamento da informação inclui pelo menos três estágios essenciais: a identificação do objeto, a ativação do nome e a generalização da resposta. Primeiro, um objeto deve ser identificado como pertencendo a uma classe particular por sua aparência ou traçado, orientação e disposição. Então, nomes apropriados devem ser ativados entre as palavras já conhecidas no léxico mental e, finalmente, deve haver uma organização fonoarticulatória para que uma resposta específica possa ser executada (Johnson; Clark; Paivio, 1996; Okada et al., 2000).

Reconhecer o significado de uma figura e nomeá-la diz respeito, em grande parte, a experiências sociais prévias e a processamentos de linguagem complexos. As vias pelas quais se processam as entradas - a auditiva e a visual - são responsáveis pela compreensão e a saída comum para todas essas vias é a fala, encarregada de dizer como a criança compreendeu e integrou as diferentes informações lingüísticas e não lingüísticas (Scheuer, Stivanin e Mangilli, 2004).

Para essas autoras, a nomeação implica e decorre de vários fatores: do desenvolvimento da memória de curto e longo prazo, da memória das propriedades dos objetos e de suas relações visuo-espaciais, da orientação, do volume, do espaço que objetos e pessoas ocupam, de suas funções, de associações fonológicosemânticas, de ordenações e categorizações.

Dessa forma, a nomeação está diretamente relacionada à memória de longo prazo, com recuperações mais permanentes (familiaridade, freqüência e concretude) e, mais especificamente, com a semântica, enquanto sua relação com a memória de curto prazo vincula-se às características estruturais de cada pa- lavra: extensão, complexidade articulatória e fonológica (Baddeley, 1986).

Essa relação, entre os atributos da figura e a memória, pode ser demonstrada no trabalho de Cycowicz et al. (1997). Segundo esses autores, palavras e figuras mais familiares e de alta freqüência no cotidiano da criança produzem respostas mais rápidas e corretas do que nomes e figuras menos familiares e de baixa freqüência. De acordo com Béfi-Lopes (1997), palavras compostas por fonemas que já fazem parte do repertório fonológico da criança são adquiridas mais rapidamente e, portanto, o controle fonológico das palavras utilizadas deve favorecer o processo de aquisição lexical.

Bee (1996) descreve que, no período inicial de aquisição de linguagem, as crianças costumam aprender palavras muito lentamente, sendo que esse padrão muda entre os $18 \mathrm{e}$ 24 meses em função da diversificação do vocabulário. Durante os anos pré-escolares, existe uma outra grande ampliação no crescimento do vocabulário. Contudo, esta se refere à velocidade, pois as crianças aprendem de cinco a dez palavras diferentes por dia. Elas conseguem isso porque parecem prestar atenção às palavras como grupos de significado ou categorias semânticas, como as que nomeiam objetos de uma única classe ou as que têm significados semelhantes.

Nelson (1996) aponta que a habilidade para reconhecer e nomear figuras emerge em um desenvolvimento contínuo que vai desde as idades mais precoces e continua a aumentar através dos anos escolares. Segundo McGregor et al. (2002), as representações lexicais ainda não estão consolidadas entre cinco e sete anos de idade e os erros na nomeação ocorrem, então, porque as crianças ainda estão em processo de organização do significado para acessar a palavra correta.

Enquanto o processo de reconhecimento e a nomeação de objetos desenvolvem-se por meio da aprendizagem espontânea e não consciente, a linguagem escrita é uma habilidade desenvolvida na aprendizagem formal e culturalmente transmitida de uma geração a outra. 
Com o intuito de explicar como a informação escrita é processada, um dos modelos desenvolvidos foi o conexionista, descrito por Adams (1991). Esse modelo é caracterizado por quatro processadores que operam interligados e em paralelo e/ou simultaneamente. 0 processador ortográfico contém as representações visuais das palavras escritas. À medida que a imagem da estrutura ortográfica vai tomando forma, sinais são enviados para as unidades do processador semântico, no qual estão armazenados os significados de palavras familiares, permitindo a compreensão da leitura. Ao mesmo tempo, uma estimulação é enviada do processador ortográfico às unidades do processador fonológico, que contém unidades correspondentes à imagem auditiva de cada palavra, sílaba ou fonema. 0 processador contextual representa o conhecimento do contexto em que o enunciado está, auxiliando na interpretação da leitura. As unidades de cada processador funcionam recebendo os sinais e enviando-os de volta ao processador de origem, facilitando o processo de reconhecimento da palavra.

Um outro modelo de leitura, conhecido como de múltiplos processamentos, concebe que a leitura é realizada por meio de um processo semântico-lexical ou fonológico (Coltheart et al., 1993; Ellis, 1995; Lecours; Parente, 1997). A rota lexical (via direta) é usada para a leitura de uma palavra armazenada, por meio da recuperação de seu significado e de sua pronúncia. Já a leitura por meio da rota fonológica (via indireta) envolve a conversão grafema-fonema para uma palavra ainda não estocada no léxico.

Baseado no modelo de Frith (1985), o desenvolvimento da leitura ocorre em três estágios: o logográfico, no qual a criança trata a palavra escrita como se fosse uma representação pictórico-ideográfica e visual do referente; o alfabético em que, com o desenvolvimento da rota fonológica, a criança aprende a fazer a decodificação grafo-fonêmica; e o ortográfico, quando, com o desenvolvimento da rota lexical, a criança aprende a fazer leitura visual direta de palavras de alta freqüência. As três estratégias ficam sempre disponíveis e a criança aprende a fazer uso daquela que se revelar mais eficaz para um ou outro tipo de material de leitura e escrita.

Salles e Parente (2002) consideram que, no inicio, o desenvolvimento da leitura é caracterizado, principalmente, pelo uso da rota fonológica. No início da $2^{\text {a }}$ série, a criança parece ainda não possuir um extenso vocabulário identificado por via direta ou sem mediação fonológica. Crianças de $3^{a}$ série já apresentam uma maior habilidade nas rotas fonológica e lexical, sugerindo uma evolução da primeira para a segunda.

Assim como a nomeação, ler uma palavra também requer conhecimento prévio de regras fonológicas e semânticas, bem como as ortográficas aplicadas ao material gráfico. Variáveis psicolingüísticas do estímulo como lexicalidade, freqüência de ocorrência, concretude, regularidade e extensão influenciam o processo de leitura (Pinheiro; Parente, 1999).

Segundo Balota; Law; Zevin (2000), palavras que ocorrem com maior freqüência durante o aprendizado de leitura e escrita são lidas mais rapidamente do que as de menor freqüência. Anteriormente, Rey et al., (1998) relataram que, além do efeito freqüente de ocorrência de palavras na leitura, palavras com menor número de grafemas são identificadas mais rapidamente do que as com maior número de grafemas.

Outro dado relevante é que, com o crescimento do vocabulário, o número de palavras acusticamente similares também aumenta, iniciando uma pressão para implementar representações fonológicas cada vez mais detalhadas e bem definidas, o que vai auxiliar, posteriormente, no processamento fonológico (Snowling, 1980; Eysenck; Keane, 1990; Troia; Roth; YeniKomshian, 1996; Swan; Goswami, 1997; Santos; Navas, 2002). Acredita-se que as habilidades de processamento fonológico, no que diz respeito à velocidade de acesso à informação fonológica no léxico mental, exercem um papel relevante na aquisição da leitura (Snowling, 1980). Assim, o tempo (latência) que a criança leva para processar uma informação visual escrita e/ou uma 
imagem pode apontar para dificuldades na nomeação e na leitura e conduz a hipóteses sobre desenvolvimento, tanto de linguagem como cognitivo.

Nessa perspectiva, o objetivo deste trabalho foi o de verificar o tempo de latência e o número de acertos para nomeação de figuras e leitura em voz alta de palavras isoladas, levando em consideração os atributos do material escrito (freqüência de ocorrência e extensão da palavra).

\section{Casuística e método}

\section{Casuística}

Os sujeitos desta pesquisa foram crianças de $2^{\text {a }}$ a $4^{\text {a }}$ séries do ensino fundamental de uma escola regular e pública da cidade de São Paulo, constituindo três grupos: 11 alunos de 2 série $(M=8: 4 a), 12$ alunos de $3^{\text {a }}$ série $(M=9: 7 a)$ e 12 alunos de $4^{\text {a }}$ série $(M=10: 6 a)$. Esses indivíduos não apresentaram alterações gerais de desenvolvimento, linguagem e escolares.

\section{Procedimentos}

Este estudo foi aprovado pela Comissão de Ética para Análise de Projetos de Pesquisa do Hospital das Clínicas da Faculdade de Medicina da Universidade de São Paulo (protocolo de pesquisa no 637/03) e os responsáveis pelas crianças assinaram o Termo de Consentimento Pós-Informação do Hospital das Clínicas da Faculdade de Medicina.

Para a seleção desses grupos com o intuito de homogeneizá-los, realizaram-se:

- Anamnese: questionário objetivo a fim de obter dados sobre intercorrências pré, peri e pósnatais, desenvolvimento neuropsicomotor, desenvolvimento auditivo, desenvolvimento de linguagem, antecedentes familiares para alterações de linguagem, aprendizagem e saúde em geral. - Questionário de Morbidade Psiquiátrica Infantil (QMPl, Almeida Filho, 1985): para indicar crianças que apresentam riscos para pa- tologia psiquiátrica, quando a pontuação é igual ou superior a 14 .

- Escala da Associação Brasileira de Institutos de Pesquisa de Mercado (ABIPEME, Mattar, 1995): teve o objetivo de avaliar o nível socioeconômico familiar.

- Questionário escolar: fechado/direto para fornecer dados sobre fracasso escolar, repetência, evasão e necessidade de escola especial.

A Anamnese, o QMPI e a escala ABIPEME foram aplicados individualmente aos pais e o Questionário Escolar foi entregue aos professores de cada série para que o respondessem.

\section{As provas}

Foram aplicadas provas de leitura e de nomeação, constituídas por estímulos equivalentes, ou seja, na prova de leitura estava escrita a palavra galo e na prova de nomeação tinha a figura de um galo. Por essa razão, as provas de leitura e de nomeação foram aplicadas em momentos diferentes para que não houvesse contaminação de uma prova em outra.

Para a elaboração das provas, foram selecionadas 32 palavras escritas balanceadas por freqüência de ocorrência e extensão da palavra para o português brasileiro. A freqüência das palavras foi determinada por sua ocorrência para crianças na faixa pré-escolar e séries iniciais, obtidas do trabalho de Pinheiro (1996). A extensão se caracterizou por palavras de quatro e seis grafemas. Todas as palavras eram regulares para a leitura e com estrutura consoante-vogal. Os grupos de palavras foram:

- oito palavras de alta freqüência com quatro grafemas: casa, galo, sapo, boné, pato, rato, vaca, vaso.

- oito palavras de alta freqüência com seis grafemas: cavalo, girafa, sapato, macaco, jacaré, menino, menina, mágico.

- sete palavras de baixa freqüência com quatro grafemas: sofá, faca, laço, maçã, fita, jipe, taça. 
- nove palavras de baixa freqüência com seis grafemas: banana, batata, tomate, cabide, camisa, coruja, cálice, casaco, pirata.

0 programa para aplicação das provas foi especialmente construído para esta pesquisa, no Departamento de Engenharia eletrônica da Universidade Estadual de Campinas (UNICAMP), por Pasquini (2004). As figuras foram elaboradas em traçado preto e fundo branco e apresentavam, em média, tamanho de $5 \mathrm{~cm} \times 5 \mathrm{~cm}$. As palavras escritas apresentavam letras impressas em negrito, fonte Microsoft Sans Serif, tamanho 40. Os estímulos apareciam um por vez no centro da tela (15 polegadas) de um computador portátil. A criança foi instruída a dizer o nome da figura na prova de nomeação de figuras e a ler a palavra escrita na prova de leitura em voz alta de palavras isoladas. Após a resposta da criança, a pesquisadora apertava uma tecla para aparecer o estímulo seguinte. Os tempos de latência foram medidos em segundos pelo programa computacional e as respostas foram gravadas e transcritas para protocolos específicos.

Foi considerado como tempo de latência o tempo entre a apresentação do estímulo e o início da resposta dada pela criança. Como acerto, considerou-se o nome correto atribuído à figura e à leitura correta da palavra, de forma ortográfica, de acordo com os critérios de Frith (1985), sendo atribuído um ponto para cada acerto e zero para erro.

\section{Resultados}

Para a análise estatística, foram utilizadas técnicas de comparações não paramétricas em função de uma baixa amostragem. Foram aplicados os testes de Friedman e Wilcoxon e de Kruskal-Wallis e Mann Whitney (Conover, 1971). 0 primeiro, aplicado na análise de dados pareados, objetivou a comparação dos grupos de estímulos em cada uma das provas e em cada uma das séries. 0 segundo teste, aplicado em análises de dados independentes, foi utilizado para comparação das séries escolares.
Foi considerado um nível de significância de $0,10(10 \%)$.

Nos gráficos 1 e 2, estão os valores referentes ao tempo de latência para a prova de leitura e de nomeação, respectivamente. Os p-valores da comparação entre os grupos de estímulos (alta freqüência/4 grafemas, alta freqüência/6 grafemas, baixa freqüência/4 grafemas e baixa freqüência/6 grafemas) nas provas de leitura e de nomeação estão na tabela 1 .

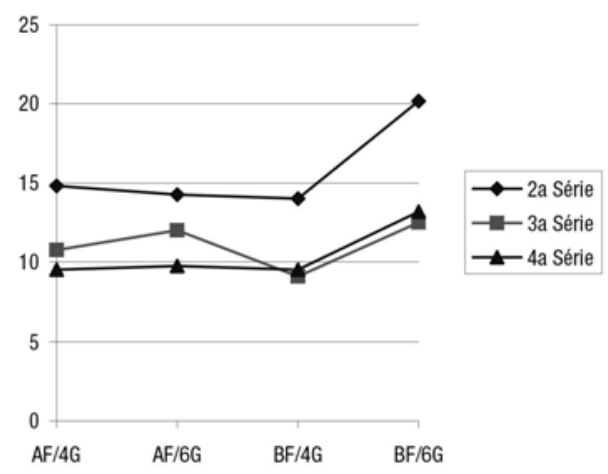

Gráfico 1. Tempo de latência, em segundos, para a leitura de palavras Legenda : $A F / 4 G$ - alta freqüência/4 grafemas; $A F / 6 G$ - alta freqüência/ 6 grafemas; BF/4G- baixa freqüência/4 grafemas; BF/6G- baixa freqüência/6 grafemas.

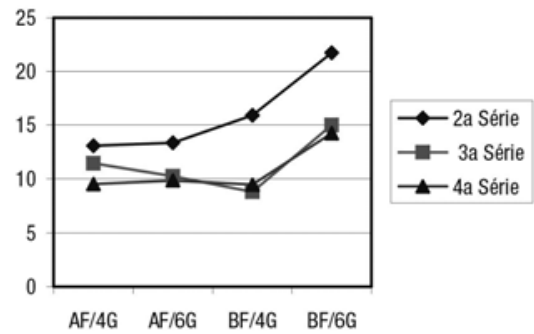

Gráfico 2. Tempo de latência, em segundos, para a nomeação de figuras Legenda : $\mathrm{AF} / 4 \mathrm{G}$ - alta frequiência/4 grafemas; $\mathrm{AF} / 6 \mathrm{G}$ - alta frequê̂ncia/6 grafemas; BF/4G- baixa freqüência/4 arafemas: BF/6G- baixa frequuência/6 arafemas.

Os resultados (tabela 1) demonstram maior tempo de latência para palavras de baixa freqüência e mais extensas. As crianças de $2^{\text {a }}$ e de $4^{\text {a }}$ séries demoraram mais tempo para acessar as palavras de baixa freqüência com seis grafemas e para nomear as figuras equivalentes. Nesses grupos de crianças, foi verificado 
Tabela 1 : p-valores para tempo de latência para leitura e nomeação de figuras

\begin{tabular}{|c|c|c|c|c|c|c|c|c|c|c|c|c|}
\hline \multicolumn{2}{|c|}{$2^{\text {a }}$ série } & $\mathrm{AF} / 4 \mathrm{G}$ & $\mathrm{AF} / 6 \mathrm{G}$ & $\mathrm{BF} / 4 \mathrm{G}$ & $3^{\mathrm{a}} \mathrm{sé}$ & & $\mathrm{AF} / 4 \mathrm{G}$ & $\mathrm{AF} / 6 \mathrm{G} \quad \mathrm{BF} / 4 \mathrm{G}$ & $4^{\mathrm{a}}$ sér & & $\mathrm{AF} / 4 \mathrm{G}$ & $\mathrm{AF} / 6 \mathrm{G} \quad \mathrm{BF} / 4 \mathrm{G}$ \\
\hline \multirow{3}{*}{ Leitura } & $\mathrm{AF} / 6 \mathrm{G}$ & 0,671 & & & \multirow{3}{*}{ Leitura } & \multicolumn{2}{|c|}{ AF/6G $\quad 0,285$} & & \multirow{3}{*}{ Leitura } & \multicolumn{3}{|c|}{ AF/6G 0,334} \\
\hline & $\mathrm{BF} / 4 \mathrm{G}$ & \multicolumn{3}{|l|}{0,859} & & \multicolumn{3}{|c|}{$\mathrm{BF} / 4 \mathrm{G} \quad 0,003^{*}$} & & \multicolumn{3}{|c|}{$\mathrm{BF} / 4 \mathrm{G} \quad 0,916$} \\
\hline & $\mathrm{BF} / 6 \mathrm{G}$ & & $0,003^{\star}$ & $0,005^{\star}$ & & $\mathrm{BF} / 6 \mathrm{G}$ & & $0,004^{\star} 0,003^{\star}$ & & $\mathrm{BF} / 6 \mathrm{G}$ & & $0,003^{\star} \quad 0,003^{\star}$ \\
\hline \multirow{3}{*}{ Nomeação } & $\mathrm{AF} / 6 \mathrm{G}$ & 0,304 & & & \multicolumn{4}{|c|}{ AF/6G 0,324} & \multicolumn{4}{|c|}{ AF/6G 0,797} \\
\hline & $\mathrm{BF} / 4 \mathrm{G}$ & 0,779 & & & Nomeaçãa & $\mathrm{BF} / 4 \mathrm{G}$ & $0,092^{\star}$ & & eeação & $\mathrm{BF} / 4 \mathrm{G}$ & 0,751 & \\
\hline & $\mathrm{BF} / 6 \mathrm{G}$ & & $0,004^{*}$ & $0,050^{*}$ & & $\mathrm{BF} / 6 \mathrm{G}$ & & $0,003^{\star} 0,003^{\star}$ & & $\mathrm{BF} / 6 \mathrm{G}$ & & $0,018^{\star} \quad 0,018^{\star}$ \\
\hline
\end{tabular}

Legenda : $\quad$ AF/4G- alta frequêencia/4 grafemas; $\mathrm{AF} / 6 \mathrm{G}$ - alta freqüência/6 grafemas;

$\mathrm{BF} / 4 \mathrm{G}$ - baixa freqüência/4 grafemas; $\mathrm{BF} / 6 \mathrm{G}$-baixa freqüência/6 grafemas.

$\mathrm{BF} / 4 \mathrm{G}$ - baixa freaüência/4 arafemas: $\mathrm{BF} / 6 \mathrm{G}$-baixa freaüência/6 arafemas.

Tabela 2 : p-valores para tempo de latência - comparação entre séries nas provas de leitura e nomeação

\begin{tabular}{|c|c|c|c|c|c|c|c|}
\hline & & $2^{\mathrm{a}}$ série & $3^{4}$ série & \multicolumn{2}{|c|}{ Nomeação } & \multirow{2}{*}{$\begin{array}{c}2^{2} \text { série } \\
0,176\end{array}$} & \multirow[t]{2}{*}{$3^{a}$ série } \\
\hline \multirow[b]{2}{*}{$\mathrm{AF} / 4 \mathrm{G}$} & $3^{2}$ série & $0,045^{\star}$ & & \multirow[b]{2}{*}{$\mathrm{AF} / 4 \mathrm{G}$} & \multirow{2}{*}{$\frac{3^{2} \text { série }}{4^{2} \text { série }}$} & & \\
\hline & $4^{2}$ série & $0,019^{*}$ & 0,89 & & & $0,036^{*}$ & 0,571 \\
\hline \multirow[b]{2}{*}{$\mathrm{AF} / 6 \mathrm{G}$} & $3^{3}$ série & $0,054^{\star}$ & & \multirow[b]{2}{*}{$\mathrm{AF} / 6 \mathrm{G}$} & $3^{3}$ série & $0,033^{*}$ & \\
\hline & $4^{2}$ série & $0,031^{\star}$ & 0,857 & & $4^{3}$ série & $0,017^{\star}$ & 0,352 \\
\hline \multirow[t]{2}{*}{$\mathrm{BF} / 4 \mathrm{G}$} & $3^{3}$ série & $0,017^{\star}$ & & \multirow[t]{2}{*}{$\mathrm{BF} / 4 \mathrm{G}$} & $3^{3}$ série & $0,001^{\star}$ & \\
\hline & $4^{2}$ série & $0,107 \#$ & 0,401 & & $4^{2}$ série & $0,006^{*}$ & 0,952 \\
\hline \multirow[t]{2}{*}{$\mathrm{BF} / 6 \mathrm{G}$} & $3^{2}$ série & $0,045^{\star}$ & & \multirow[t]{2}{*}{$\mathrm{BF} / 6 \mathrm{G}$} & $3^{2}$ série & $0,001^{*}$ & \\
\hline & $4^{2}$ série & $0,037^{\star}$ & 1 & & $4^{2}$ série & $0,009^{*}$ & 0,582 \\
\hline
\end{tabular}

Legenda: $A F / 4 G-$ alta freqüência/4 grafemas; AF/6G- alta freqüência/6 grafemas;

BF/4G- baixa freaüência/4 arafemas: BF/6G-baixa freaüência/6 arafemas.

efeito de freqüência para palavras com seis grafe-mas e efeito de extensão para palavras de baixa freqüência. 0 efeito de freqüência ocorreu também nas crianças de $3^{\text {a }}$ série para quatro e seis grafemas, enquanto o efeito de extensão se mostrou somente para palavras e figuras com baixa freqüência, determinando um maior tempo de latência.

$\mathrm{Na}$ tabela 2, podem ser visualizados os $p$-valores da comparação entre as séries escolares para o tempo de latência.

De acordo com os dados da tabela 2 , verificou-se diferença estatisticamente significante entre a $2^{\text {a }}$ e as outras séries, para todos os grupos de estímulos, em ambas as provas. $\mathrm{Na}$ comparação entre a $3^{\mathrm{a}}$ e $4^{\mathrm{a}}$ séries, não foi verificada diferença estatisticamente significante. A média do tempo de latência para $3^{\text {a }}$ e $4^{\text {a }}$ séries foi semelhante, enquanto as crianças de $2^{a}$ série levaram mais tempo para ler palavras e nomear figuras. Esses dados também podem ser visualizados nos gráficos 1 e 2 .
Nos gráficos 3 e 4, estão os valores referentes aos acertos para a prova de leitura e de nomeação. Na tabela 3, estão os p-valores da comparação entre os grupos de estímulos para ambas as provas.

A análise estatística (tabela 3) mostrou, quanto aos acertos, que não ocorreu efeito de freqüência e de extensão para a leitura na $2^{\text {a }}$ série. Nas crianças de $3^{\mathrm{a}}$ e $4^{\mathrm{a}}$ séries, houve efeito de freqüência para as palavras com seis grafemas (entre as palavras com seis grafemas, as de alta freqüência são lidas mais corretamente do que as de baixa). 0 efeito de extensão foi observado apenas para palavras de baixa freqüência na $3^{\text {a }}$ série (entre as palavras de baixa freqüência, aquelas com quatro grafemas tiveram mais acertos).

Na prova de nomeação, foi observado efeito de freqüência para o grupo de figuras equivalentes às palavras com quatro e seis grafemas nas três séries. 0 efeito de extensão foi observado somente nos grupos de figuras equivalentes às palavras menos freqüentes para $3^{\mathrm{a}}$ e $4^{\mathrm{a}}$ séries. 


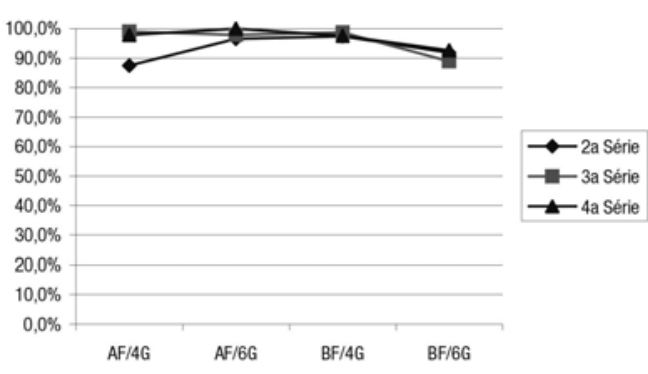

Gráfico 3 . Acertos para a leitura de palavras

Legenda : AF/4G-alta frequêencia/4 grafemas; AF/6G-alta frequêencia/6 grafemas; $\mathrm{BF} / 4 \mathrm{G}$ - baixa freqüência/4 grafemas; $\mathrm{BF} / 6 \mathrm{G}$ - baixa freqüência/ 6 grafemas.

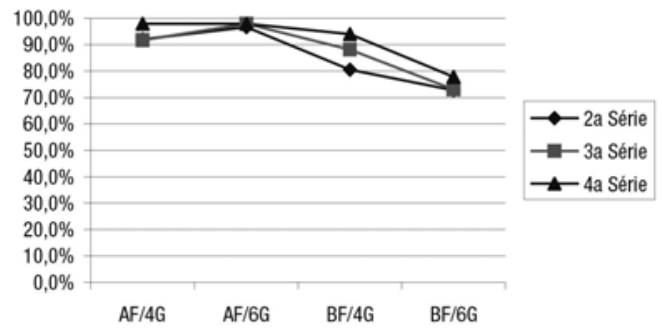

Gráfico 4 . Acertos para a nomeaçăo de figuras

Legenda : AF/4G-alta freqüêencia/4 grafemas; AF/6G-alta frequeência/6 grafemas; $\mathrm{BF} / 4 \mathrm{G}$ - baixa frequêência/4 grafemas; BF/6G- baixa frequêência/6 grafemas.

Tabela 3 : p-valores para acertos na leitura de palavras e nomeação de figuras

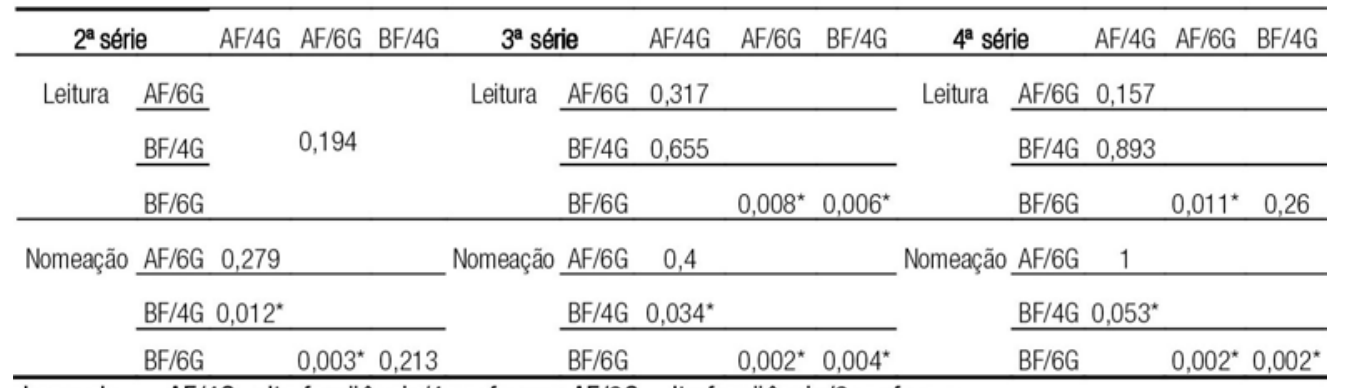

Legenda : $\quad \mathrm{AF} / 4 \mathrm{G}$ - alta freqüência/4 grafemas; $\mathrm{AF} / 6 \mathrm{G}$ - alta freqüência/6 grafemas;

BF/4G- baixa freaüência/4arafemas: BF/6G-baixa freaüência/6 arafemas.

Tabela 4: p-valores para acertos - comparação entre séries nas provas de Leitura e Nomeação

\begin{tabular}{|c|c|c|c|c|c|c|c|}
\hline \multicolumn{2}{|c|}{ Leitura } & $2^{n}$ série & $3^{n}$ série & \multicolumn{2}{|c|}{ Nomeação } & $2^{2}$ série & $3^{3}$ série \\
\hline \multirow{2}{*}{$\mathrm{AF} / 4 \mathrm{G}$} & $3^{2}$ série & \multirow{2}{*}{\multicolumn{2}{|c|}{0,423}} & \multirow[b]{2}{*}{$\mathrm{AF} / 4 \mathrm{G}$} & $3^{2}$ série & \multicolumn{2}{|l|}{0,891} \\
\hline & $4^{2}$ série & & & & $4^{3}$ série & $0,055^{*}$ & $0,035^{*}$ \\
\hline \multirow{2}{*}{$\mathrm{AF} / 6 \mathrm{G}$} & $3^{2}$ série & \multirow{2}{*}{\multicolumn{2}{|c|}{0,138}} & $\mathrm{AF} / 6 \mathrm{G}$ & $3^{n}$ série & \multirow{2}{*}{\multicolumn{2}{|c|}{0,977}} \\
\hline & $4^{2}$ série & & & & $4^{2}$ série & & \\
\hline \multirow[t]{2}{*}{$\mathrm{BF} / 4 \mathrm{G}$} & $3^{2}$ série & \multirow{2}{*}{\multicolumn{2}{|c|}{0,759}} & \multirow[t]{2}{*}{$\mathrm{BF} / 4 \mathrm{G}$} & $3^{3}$ série & 0,137 & \\
\hline & $4^{2}$ série & & & & $4^{2}$ série & $0,015^{\star}$ & 0,623 \\
\hline \multirow[t]{2}{*}{$\mathrm{BF} / 6 \mathrm{G}$} & $3^{2}$ série & \multirow{2}{*}{\multicolumn{2}{|c|}{0,823}} & $\mathrm{BF} / 6 \mathrm{G}$ & $3^{2}$ série & \multirow{2}{*}{\multicolumn{2}{|c|}{0,551}} \\
\hline & $4^{2}$ série & & & & $4^{3}$ série & & \\
\hline
\end{tabular}

Legenda : AF/4G- alta freqüência/4 grafemas; $A F / 6 G$ - alta frequiência/6 grafemas; BF/4G- baixa freaüência/4arafemas: BF/6G-baixa freaüência/6 arafemas. 
A comparação entre as séries escolares quanto aos acertos encontra-se na tabela 4 .

De acordo com os dados da tabela acima, não foi observada diferença significativa entre as séries escolares para a leitura, ou seja, as crianças das três séries escolares apresentaram mesmo percentual de acertos. Na nomeação, as crianças de $4^{\mathrm{a}}$ série acertaram mais do que as de $2^{\text {a }}$ e as de $3^{\text {a }}$ ao nomearem figuras equivalentes às palavras de alta freqüência/4 grafemas. Também acertaram mais do que as de $2^{\text {a }}$ para nomearem o grupo equivalente às palavras de baixa freqüência/4 grafemas.

\section{Discussão}

De forma geral, observa-se influência da freqüência de ocorrência e da extensão do material escrito, tanto na exatidão da leitura quanto no tempo gasto para iniciar a leitura das palavras.

Quanto ao tempo de latência, foi observado que crianças de $2^{\mathrm{a}}, 3^{\mathrm{a}}$ e $4^{\mathrm{a}}$ séries levam mais tempo para acessar as respostas quando as palavras são menos freqüentes e mais extensas.

A influência de variáveis psicolingüísticas na leitura de palavras está de acordo com as pesquisas realizadas por Rey et al. (1998), Balota; Law; Zevin (2000) e Salles; Parente (2002). Como as palavras de alta freqüência repetem-se muitas vezes e são mais facilmente reconhecidas, tendem a produzir um menor tempo de latência e produzem menos erros. Da mesma forma, quanto maior a extensão da palavra a ser lida, maior a sobrecarga na memória de trabalho fonológica, incorrendo mais imprecisões na leitura e maior tempo de latência (Baddeley, 1998).

0 tempo de latência para iniciar a leitura das palavras diminui com a escolarização, demonstrando desenvolvimento e uma maior eficiência na tarefa. Pesquisar esse tempo é importante, pois o acesso preciso e rápido ao léxico mental influencia no tempo gasto na leitura de um texto e, conseqüentemente, em sua compreensão (Salles; Parente, 2002). Apesar de ter ocorrido o efeito de freqüência e de extensão para o tempo de latência na leitura, as respostas das crianças mais novas ( $2^{\text {a }}$ série) não foram afetadas pelo tipo de palavra escrita quanto aos acertos. Essas crianças leram de forma ortográfica palavras de freqüência e extensão variáveis, porque utilizam preferencialmente a rota fonológica, a que permite a realização da leitura por meio de conversão grafofonêmica (Salles; Parente, 2002). Se as crianças mais novas tivessem sido expostas à leitura de palavras com estruturas mais complexas e ortografia irregular (neste estudo foram incluídas apenas palavras com estrutura silábica do tipo consoante-vogal e com ortografia regular), provavelmente apresentariam menos acertos nesse grupo de palavras, seriam mais imprecisas. Isso porque a leitura de palavras irregulares é realizada por meio da rota lexical que ainda não está desenvolvida no início da escolarização (Coltheart et al., 2001).

As crianças de $3^{\text {a }}$ e $4^{\text {a }}$ séries também leram de forma ortográfica, mas sua leitura é facilitada quando as palavras são mais freqüentes e menos extensas. Como as crianças nessas séries já apresentam habilidades semelhantes para as rotas fonológica e lexical, elas podem ler tanto por meio de conversão grafofonêmica quanto por meio do acesso ao significado da palavra (Salles; Parente, 2002).

As autoras acima citadas apontam, ainda, que os leitores preferencialmente lexicais podem ter uma leitura mais rápida pela auto-maticidade do acesso ao léxico, mas podem apresentar sérias dificuldades na leitura de palavras menos freqüentes. Esse fato foi observado na leitura por crianças de $3^{\text {a }}$ e $4^{\text {a }}$ séries, quando apresentaram maior tempo de latência e mais imprecisões ao lerem palavras menos freqüentes.

Quanto à nomeação de figuras, as crianças de $2^{\mathrm{a}}$ a $4^{\mathrm{a}}$ séries apresentaram maior percentual de respostas corretas e menor tempo de latência para figuras referentes às palavras de menor freqüência e extensão. Esse comportamento, semelhante ao da leitura de palavras, aponta vias comuns aos processamentos de pa- 
lavras escritas e de figuras. De acordo com Bajo (1998), figuras e palavras são representadas por um sistema semântico comum e as diferenças são explicadas pelo acesso aos diferentes tipos de características do estímulo, ou seja, há códigos visuais mais elaborados para figuras quando comparadas às palavras ou, ainda, maior distinção das representações semânticas para figuras em relação às palavras.

Nomeação de figuras e leitura de palavras requerem a recuperação da informação fonológica (Snowlling, 1991) e semântica (Bajo, 1998) da memória de longo prazo. Aspectos semânticos (familiaridade, freqüência e concre-tude) e fonológicos (extensão e complexidade articulatória) devem ser organizados na memória e acessados frente às particularidades de um estímulo.

Somente as crianças de $2^{\text {a }}$ série apresentaram diferenças entre os grupos de estímulos na nomeação, não ocorrendo o mesmo para a leitura das palavras. Como já foi explicado acima, a leitura na $2^{\text {a }}$ série pode ocorrer sem o acesso à palavra como um todo e a seu significado, levando a um comportamento indiferenciado para todos os tipos de estímulos. Já a nomeação envolve a recuperação do significado e a seleção do nome correto para designar a figura (Johnson; Clark; Paivio, 1996).
Esse processo já sofre, então, influência da freqüência e extensão do estímulo.

Embora este estudo tenha sido com um grupo piloto, os dados obtidos apontam que as crianças se comportam diferentemente quando submetidas a estímulos com determinadas características. Além disso, elas se tornam mais eficientes com a escolarização e com a idade, demonstrando aprendizagem para estímulos com diferentes propriedades, semânticas e fonológicas.

\section{Conclusão}

- Em relação à comparação intra-série para leitura, houve influência das variáveis do material escrito, sendo palavras de alta freqüência e de menor extensão acessadas de forma mais precisa por crianças de $3^{\text {a e }} 4^{\text {a }}$ séries. Quanto ao tempo de latência, crianças de $2^{\text {a }}$ a $4^{\mathrm{a}}$ séries acessaram mais rapidamente palavras mais freqüentes e menos extensas.

- A nomeação de figuras foi um processo que se assemelhou à leitura de palavras por influência de variáveis psicolingüísticas no tempo de latência e nos acertos.

- Com a idade e escolarização, as crianças tornam-se mais rápidas para iniciar a leitura de palavras isoladas e para nomear figuras equivalentes.

\section{Referências bibliográficas}

ADAMS, M. J. Beginning to read: thinking and learning about print. Massachusetts: Massachusetts Institute of Technology, 1991.

ALMEIDA FILHO, N. Epidemiologia das desordens mentais da infância no Brasil. Salvador: Centro Editorial e Didático da Universidade Federal da Bahia, 1985.

BADDELEY, A. Working memory. Oxford: Oxford University Press, 1986.

The human memory: theory and practice. Boston: Allyn and Bacon, 1998.

BALOTA, D. A.; LAW, M. B.; ZEVIN, J. D. The attentional control of lexical processing pathways: Reversing the word frequency effect. Memory \& Cognition, v. 28 n.7, p. 1081-1089, 2000.

BAJO, M. Semantic facilitation with pictures and words. Journal of Experimental Psychological: Learning, Memory and Cognition, v. 14, p. 579-589, 1998. 
BEE, H. A criança em desenvolvimento. $7^{\text {a }}$ ed., Porto Alegre: Artes Médicas, 1996.

BÉFI-LOPES, D. M. Aspectos da competência e do desempenho lexicais em crianças entre 4:0 e 6:6 anos, com padrões de desenvolvimento normal de linguagem e com alterações articulatórias. Tese (Doutorado), Universidade de São Paulo. 1997.

COLTHEART, M. et al. Models of reading aloud: dual route and parallel - distributed - processing approaches. Psychological Review, v.100, p. 589-608, 1993.

COLTHEART, M. et al. DRC: a dual route cascated model of visual word recognition and reading aloud. Psychological Review, v.108, p. 204-256, 2001.

CONOVER, W. U. Pratical nonparametric statistics, $1^{\text {a }}$ ed. New York: Jonh Willy \& Sons, 1971.

CYCOWICZ, Y. M. et al. Picture naming by young children: norms for name, agreement, familiarity and visual complexity. Journal of Experimental Child Psychological, v. 65, p. 171-237, 1997.

ELLIS, A. W. Leitura e escrita e dislexia: uma análise cognitiva. 2ª ed, Artes Médicas, Porto Alegre, 1995.

EYSENCK, M. W.; KEANE, M. T. Cognitive psychology: a student's handbook. New York: Lawrence Erlbaum, 1990.

FRITH, U. Beneath the surface of developmental dyslexia. In: Patterson, K.; Marshall, J.; Colthearts M. (Eds.) Surface dyslexia: neuropsychological and cognitive studies of phonological reading. London: Lawrence Erlbaum Associates, 1985.

JOHNSON, C. J.; CLARK, J. M.; PAIVIO, A. Cognitive components of picture naming. Psychological Bulletin, v. 120, n. 1, p. 113139,1996

LECOURS, A. R.; PARENTE, M. A. M. P. Dislexia: implicações do sistema de escrita do português. São Paulo: Artes Médicas, 1997.

MATTAR, F. N. Análise crítica dos estudos de estratificação sócio-econômica de ABA-Abipeme. Revista de Administração. São Paulo v.30, n.1, p. 57-74, janeiro/março 1995.

McGREGOR, K. K. et al. Semantic representation and naming in young children. Journal of Speech, Language and Hearing Research, v.45, p. 332-346, 2002.

NELSON, K. Language in cognitive development: emergence of the mediated mind. New York: Cambridge University Press, 1996.

OKADA, T. et al. Naming of animal and tool: a functional magnetic resonance imaging study of categorical differences in the human brain areas commonly used for naming visually presented objects. Neuroscience Letter, v. 296, p. 33-36, 2000.

PINHEIRO, A. M. V. Contagem de freqüência de ocorrência de palavras expostas a crianças na faixa de pré-escola e séries iniciais. Software produzido pela Associação Brasileira de Dislexia - ABD, 1996.

PINHEIRO, A. M. V.; PARENTE, M. A. M. P. Estudo de caso de um paciente com dislexia periférica e as implicações dessa condição nos processamentos centrais. Pró-Fono Revista de Atualização Científica, v. 11, n. 1, p. 115-123, 1999.

REY, A. et al. A phoneme effect in visual word recognition. Cognition, v. 68, p.71-80, 1998.

SALLES, J. F.; PARENTE, M. A. M. P. Relação entre os processos cognitivos envolvidos na leitura de palavras e as habilidades de consciência fonológica em escolares. Pró-Fono, Carapicuíba -SP, v. 14, n. 2, p. 175-186, maio/ago., 2002.

SANTOS, M. T. M.; NAVAS, A. L. G. P. Aquisição e desenvolvimento da linguagem escrita. In: Distúrbios de leitura escrita: teoria e prática. Ed. Manole, 2002. 
SCHEUER, C. I.; STIVANIN, L.; MANGILLI, L. D. Nomeação de figures e a memória em crianças: efeitos fonológicos e semânticos. Pró-Fono Revista de Atualização Científica, Barueri (SP), v. 16, n. 1, p. 49-56, jan./abr., 2004.

SNOWLING, M. J. Object Naming deficits in developmental dyslexia. Journal of Research in Reading, v. 11, p. 67-85, 1980.

SWAN, D.; GOSWAMI, U. Picture naming deficits in developmental dyslexia: the phonological representations hypothesis. Brain and Language, v. 56, p. 334-353, 1997.

TROIA, G. A.; ROTH, F. P.; YENI-KOMSHIAN, G. H. Word frequency and age effects in normally developing children's phonological processing. Journal of Speech and Hearing Research, v. 39, p. 1099-1108, 1996.

Recebido em 27.09.04

Modificado em 29.06.05

Aprovado em 20.07.05

Luciene Stivanin é fonoaudióloga especializada em Processo Terapêutico nas Alterações Sensório-Motores de Origens Sindrômica e Não-Sindrômica no Curso de Fonoaudiologia, Departamento de Fisioterapia, Fonoaudiologia e Terapia Ocupacional da Faculdade de Medicina da Universidade de São Paulo. Mestranda em Ciências: Programa de Pós-Graduação - Fisiopatologia Experimental da FMUSP. Supervisora do Laboratório de Investigação Fonoaudiológica em Linguagem Infantil e Cognição do Curso do Fonoaudiologia da FMUSP.

Claudia Inês Scheuer é fonoaudióloga e doutora em Lingüistica. Docente do Curso de Fonoaudiologia, Departamento de Fisioterapia, Fonoaudiologia e Terapia Ocupacional da FMUSP. 